\title{
Coherent frequency division with a degenerate synchronously pumped optical parametric oscillator
}

\author{
Chenchen Wan, ${ }^{1,2,+}$ Peng LI, ${ }^{1, *}$ Axel Rueht, ${ }^{1,}$ And Ingmar Harti ${ }^{1,}{ }^{*}$ \\ ${ }^{1}$ Deutsches Elektronen-Synchrotron (DESY), Notkestraße 85, 22607 Hamburg, Germany \\ ${ }^{2}$ Electro-optics Program, University of Dayton, 300 College Park, Dayton, $\mathrm{OH}, 45469-0245$, USA \\ ${ }^{+}$Current affiliation: Thorlabs Inc., 56 Sparta Ave., Newton, NJ, USA \\ ${ }^{\ddagger}$ Current affiliation: IMRA America Inc., 1044 Woodridge Ave., Ann Arbor, MI 48105, USA \\ ${ }^{\S}$ Current affiliation: Leibniz University Hannover, QUEST-Leibniz-Research School, Institut for Quantum Optics, Welfengarten 1, 30167 \\ Hannover, Germany \\ *Corresponding author: ingmar.hartl@desy.de \\ Received XX Month XXXX; revised XX Month, XXXX; accepted XX Month XXXX; posted XX Month XXXX (Doc. ID XXXXX); published XX Month \\ $X X X X$
}

Synchronously-pumped optical parametric oscillators (OPOs) are important tools for frequency comb (FC) generation in the mid-IR spectral range, where few suitable laser gain materials exist. For degenerate OPOs self-phase-locking to the pump FC has been demonstrated. Here, we present a phase noise study of the its carrier envelope offset frequency, revealing a -6 dB reduction compared to the pump frequency comb over a wide Fourier frequency range. These results demonstrate that a degenerate optical parametric oscillator can be an ideal coherent frequency divider without any excess noise.

\section{(C) 2017 Optical Society of America}

OCIS codes: (190.4970) Parametric oscillators and amplifiers; (190.4975) Parametric process; (270.2500) Fluctuations, relaxations, and noise;

http://dx.doi.org/10.1364/OL.99.099999

Optical frequency combs (FC) consisting of equally spaced frequency modes have enabled advances in numerous applications in the field of optical metrology [1] and spectroscopy [2]. There is a growing demand of extending the spectral range of FCs to the mid-IR $(2-20 \mu \mathrm{m})$ and molecular fingerprint region, respectively where mid-IR FC spectroscopy can be used to understand molecular structures or to perform gas analysis for biological and environmental applications [3]. Direct generation of mid-IR FCs has been reported using mode-locked lasers based on novel gain media, quantum cascade laser technology [4] and micro-resonator based Kerr comb [5], nonlinear frequency conversion process such as difference frequency generation [6,7] and optical parametric oscillation [8] still have the advantages of a mature technology platform enabling a wide wavelength coverage.
Optical parametric oscillators (OPOs) synchronously pumped by a femtosecond FC have been used to generate FCs in both single [9] and doubly resonant [10] operation regimes. In doubly resonant OPOs, the oscillation occurs at discrete cavity lengths ("resonances"), separated by $\lambda / 2$, where $\lambda$ is the center wavelength of the OPO. When operated at non-degeneracy [11], the OPO output spectrum is tunable. Here, the output spectrum consists of two combs for the signal- and idler- field respectively. Their respective carrier envelope offset frequency $f_{0}$ is not fixed to the driving FC, requiring an additional active feedback loop for stabilization. On the other hand, degenerate OPOs (DOPO) have shown to produce a sub-harmonic of the driving FC. Here the subharmonic OPO field is self-phase-locked to the pump [12-16]. Although a dither-lock of the OPO cavity length is usually applied to keep the DOPO long-term stable at a cavity resonance, the selfphase-locking of DOPOs combs to the pumb combs has been observed even without active stabilization [12]. The frequency comb lines of DOPOs can only take two sets of modes shifted by $f_{\text {rep, }}$ $p / 2$, where $f_{\text {rep }, p}$ is the pump repetition frequency [15]. The DOPO comb alternates between those two sets when tuning from one cavity resonance to the next. While the DOPO comb's repetition frequency is always equal to the pump FC's $f_{\text {rep, } p}$, its offset frequency alternates between $f_{0, p} / 2$ and $f_{0, p} / 2+f_{\text {rep, }} / 2$, where $f_{0, p}$ is the pump offset frequency. In both cases the DOPO comb is selflocked to the pump FC.

Understanding the phase noise transfer of DOPOs has been a focus of various studies. Initially the self-locking of $f_{0}$ has been verified by a stable beat note of DOPO with a reference cw laser [12]. A recent phase noise study directly measured the $f_{0}$ of a DOPO by beating the outputs parasitic sum frequency with the pump. A phase noise comparison of the pump and the DOPO $f_{0}$ indicates self-locking [16]. Here, the DOPOs $f_{0}$ phase noise power spectral density exceeds the pump lasers', with the exception of a small Fourier frequency range around $10 \mathrm{kHz}$. 
However, an early study by Nabors et al. on single-frequency DOPOs, could show a $6 \mathrm{~dB}$ reduction in modulation sidebands with respect to the modulated pump field [19]. As expected from an ideal times-n frequency multiplier, where phase noise scales with $\mathrm{n}^{2}$. This $6 \mathrm{~dB}$ reduction did hold for low modulation frequencies, until the lifetime of the DOPO cavity was further damped. For typical cavity lifetimes the on-set of this effect is observed at modulation frequencies in the MHz range.

In this letter, we study the $f_{0}$ coherence of a free-running DOPO by measuring the phase noise transfer function from the driving $\mathrm{FC}$ to the DOPO field. Our results prove for the first time, to the best of our knowledge, that the DOPO has a $f_{0}$ phase noise $6 \mathrm{~dB}$ lower than the driving FC in the Fourier frequency range from $1 \mathrm{kHz}$ to $\sim 200$ $\mathrm{kHz}$, showing strong phase-noise suppression up to several $\mathrm{MHz}$ Fourier frequencies. The noise reduction indicates that DOPO pumped by fs-FCs can serve as a coherent frequency divider just as their single-frequency counterparts.

The experimental setup sketched in Fig. 1 consists of a doubly resonant OPO based on a $1 \mathrm{~mm}$ long MgO:PPLN crystal with a poling period of $31.245 \mu \mathrm{m}$ placed in a bowtie cavity. The OPO is pumped through a dichroic mirror exhibiting a reflection greater than $99.8 \%$ of the OPO field. One of the cavity mirrors is mounted on a piezo stage for cavity length control. The driving FC is a Yb:fiber based system consisting of a $150 \mathrm{MHz}$ Yb:fiber oscillator and chirped-pulse amplifier delivering $100 \mathrm{fs}$ pulses with up to $13 \mathrm{~nJ}$ pulse energy [20]. The FC repetition rate is stabilized by phase-locking a single comb line to a single-frequency nonplanar ring oscillator at $1064 \mathrm{~nm}$ with feedback to the oscillator cavity length. The $f_{0}$ is detected with an inline f-2f interferometer and phase-locked to an RF reference by feedback to the current of the oscillator pump diode. The phase-stabilized pulse train passes an additional acousto-optic modulator (AOM) allowing to phasemodulate $f_{0}$ of the pump $\mathrm{FC}$ for the transfer function measurements.

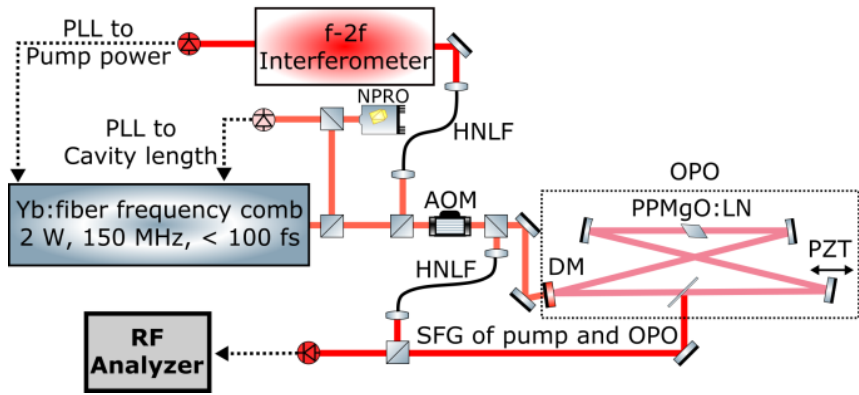

Fig. 1. Experimental setup. PLL: phase lock loop; PZT: piezo stage; AOM: acousto-optic modulator; HNLF: highly nonlinear fiber; DM: dichroic mirror, NPRO: single frequency laser. PLL to pump power and $\mathrm{AOM}$ are the two frequency modulation methods for OPO pump comb.

The $f_{0}$ of the OPO output is obtained by beating the parasitic sumfrequency signal of pump and OPO field at a wavelength of $700 \mathrm{~nm}$ with an additional supercontinuum (SC) branch of the pump FC generated in a suspended-core highly nonlinear fiber (HNLF) [21]. It can easily be shown that this scheme directly reveals the $f_{0}$ signal of the OPO which was further analyzed in the phase-noise and transfer function measurements described below.

When the OPO cavity length is tuned, several discrete resonances are observable which are separated by half of the OPO wavelength. When pumping the OPO 10 times above threshold, a maximum of 11 resonances could be observed. Within the central resonances, the OPO is initially oscillating at degeneracy but would jump to non-degeneracy with increasing the cavity length. This behavior is illustrated in Fig. 2 showing the optical spectrum, the OPO $f_{0}$ and the OPO output power vs. cavity length. At degeneracy, the OPO spectrum exhibits a single peak at the sub-harmonic of the pump and the OPO $f_{0}$ is self-locked and stays unchanged when tuning the cavity length [12]. The setup is sufficiently passively stable to remain in a degenerate operation for tens of minutes without cavity length stabilization. When scanning the OPO cavity length from short to long in one resonance, the system undergoes a region of unstable oscillations before non-degenerate operation can be observed. Here, the optical spectrum as well as the $f_{0}$ of the OPO are split into two branches representing signal and idler field. Since only the sum of these quantities is fixed, both fields hence exhibit a strong dependence on the cavity length. Depending on the slope of signal and idler $f_{0}$ with respect to the cavity length, selfphase locked behavior could indeed be observed [22] but a detailed phase-noise study would require the absolute stabilization of the cavity length which is currently not possible with our setup. The study and discussion presented here is hence restricted to degenerate operation commonly used for FC generation with OPOs.

(a)

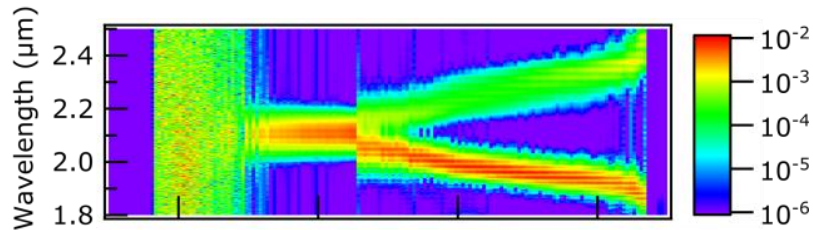

(b)

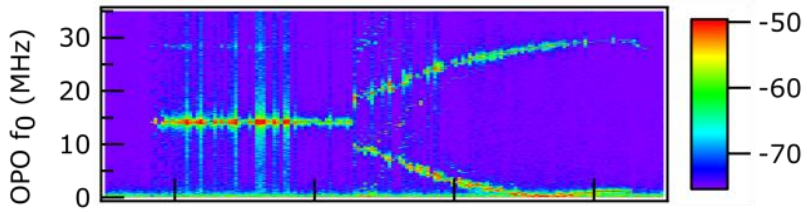

(c)

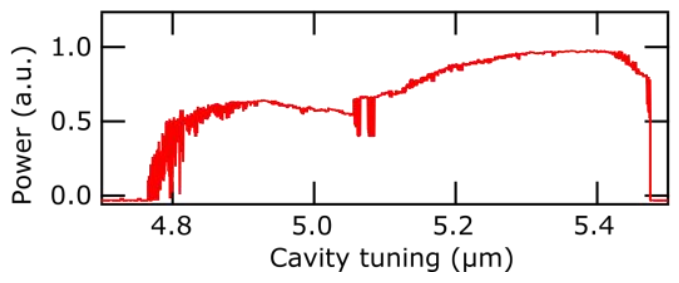

Fig. 2. Transition from degenerate to non-degenerate operation when tuning the cavity length. (a) OPO optical spectrum, (b) OPO $f_{0}$, and (c) OPO output power. Note: the output power was measured using an extended InGaAs diode, whose sensitivity is strongly wavelength dependent.

First, the phase noise of in-loop pump f-2f beating and the OPO $f_{0}$ beating were recorded simultaneously at OPO degeneracy. As shown in Fig. 3 at lower frequency $(<1 \mathrm{kHz})$ the $0 P 0$ has higher $f_{0}$ phase noise due to technical excess noise such as vibrations picked up by the unlocked OPO cavity. Over the entire $\sim 1 \mathrm{kHz}$ to $5 \mathrm{MHz}$ span, the OPO's $f_{0}$ phase noise power spectral density is lower than the pump's. A maximum reduction of $10 \mathrm{~dB}$ was observed at 10 $\mathrm{kHz}$. 


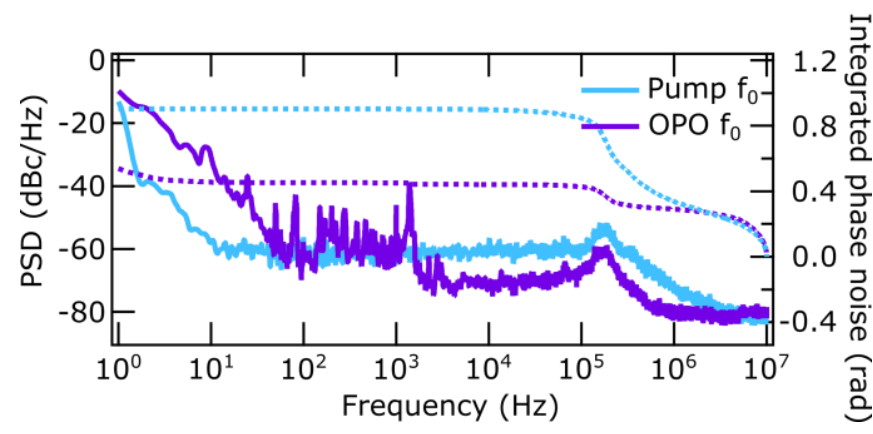

Fig. 3 Phase noise of pump and DOPO $f$. Solid lines are the RF power spectral density (PSD); dashed lines are the integrated phase noise.

Since the OPO cavity is not actively locked, any technical noise that changes the cavity length would be coupled to the phase noise. To eliminate this effect, we measure the pump to DOPO $f_{o}$ phase noise transfer function. For this measurement we phase modulate the pump's $f_{0}$ and detect the DOPO $f_{0}$ response. In our experiments we applied two different pump $f_{0}$ modulation methods.

In our first method, we add a small modulation signal to the control signal used for pump $f_{0}$ stabilization, the Yb:fiber oscillator pump diode current. The modulation frequency is scanned from $1 \mathrm{kHz}$ to $8 \mathrm{MHz}$. As shown in Fig. 4 the modulation will generate sidebands on the pump f-2f beat note as well on the DOPO $f_{0}$ beat note. For small phase modulation, the modulation index can be determined by the relative peak powers of the first sidebands to the carrier. We calculate the transfer function's value for a particular modulation frequency by taking the ratio of the modulation indices for DOPO and pump $f_{0}$.
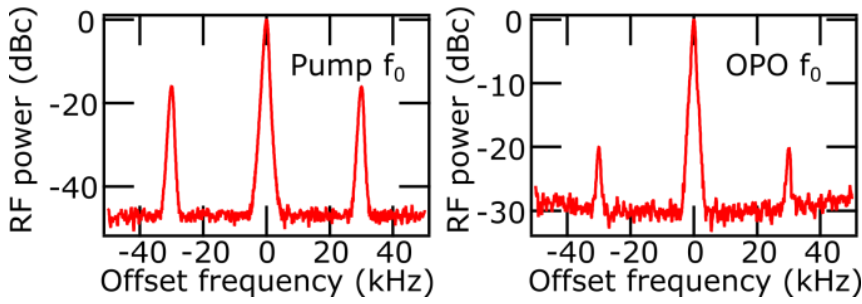

Fig. 4. The modulation sidebands on the pump and the OPO $f_{0}$ where the modulation index is determined by the sideband strength. In this plot, the modulation frequency is at $30 \mathrm{kHz}$.

Fig. 5 is a typical transfer function measurement. The modulation index of the pump $f_{0}$ increases monotonically from $1 \mathrm{kHz}$ to $\sim 50$ $\mathrm{kHz}$ then it has a dip in the range of $\sim 50 \mathrm{kHz}$ to $\sim 500 \mathrm{kHz}$ and within this range the modulation index shows large fluctuations. When the modulation frequency is above $500 \mathrm{kHz}$, the modulation index does not oscillate and monotonically decreases. The corresponding transfer function is shown in Fig. 5 . It shows a $6 \mathrm{~dB}$ modulation suppression in the range from $1 \mathrm{kHz}$ to $\sim 50 \mathrm{kHz}$. Above $500 \mathrm{kHz}$, the transfer function is negative again with a minimum of $-10 \mathrm{~dB}$. In the range of $\sim 50 \mathrm{kHz}$ to $\sim 500 \mathrm{kHz}$ the DOPO $f_{0}$ has stronger sidebands than the pump which could be interpreted as a larger DOPO $f_{0}$ phase noise than the pump. However, it turns out that the strong feature in the modulation frequency range from $50 \mathrm{kHz}$ to $\sim 500 \mathrm{kHz}$ is a the result of our modulation method. Since the modulation is added to the current of the pump diode, it modulates both amplitude and $f_{0}$ of the pump FC [23]. At low Fourier frequencies, the phase modulation is dominant and amplitude modulation can be neglected. However the pump current modulation causes large amplitude modulation in the $\sim 50 \mathrm{kHz}$ to $\sim 500 \mathrm{kHz}$ as it drives the relaxation oscillation of the laser gain material. In our measurement, only pump $f_{0}$ phase modulation is considered, thus the transfer function measurement becomes invalid around the relaxation oscillation frequency.

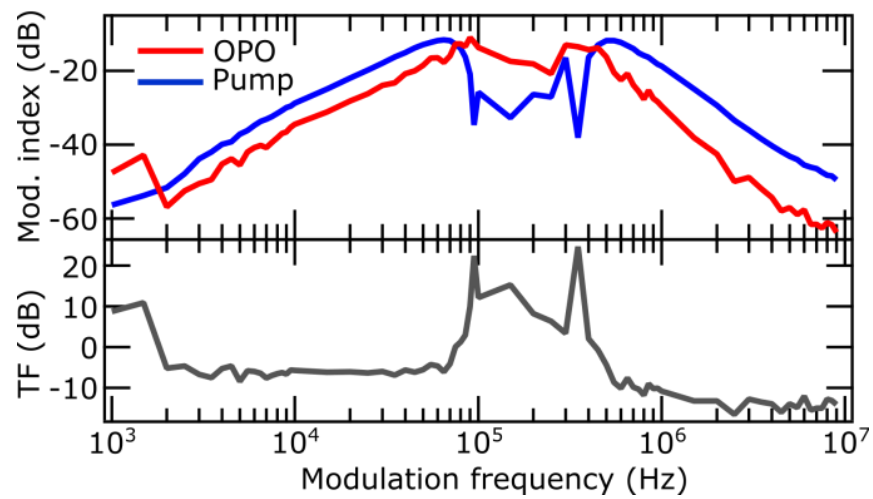

Fig. 5. Transfer function (TF) measured with pump diode modulation method. Top: modulation index of the modulation of pump and DOPO $f_{0}$ beats. The ratio of DOPO and pump modulation indices gives the $f_{0}$ noise transfer function.

To overcome this limitation and to confirm the problems with the pump power modulation discussed above, we applied a second pump $f_{0}$ phase modulation method by implementing a resonant acousto-optic modulator (AOM) after our $\mathrm{Yb}-\mathrm{FC}$ and using the diffracted beam to pump the OPO. We add a small frequency modulation to the driving RF of the AOM. The AOM will frequency shift all comb lines so it is effectively a shift of the $f_{0}$ of the driving FC. We experimentally confirmed that this method does not produce any amplitude modulation.

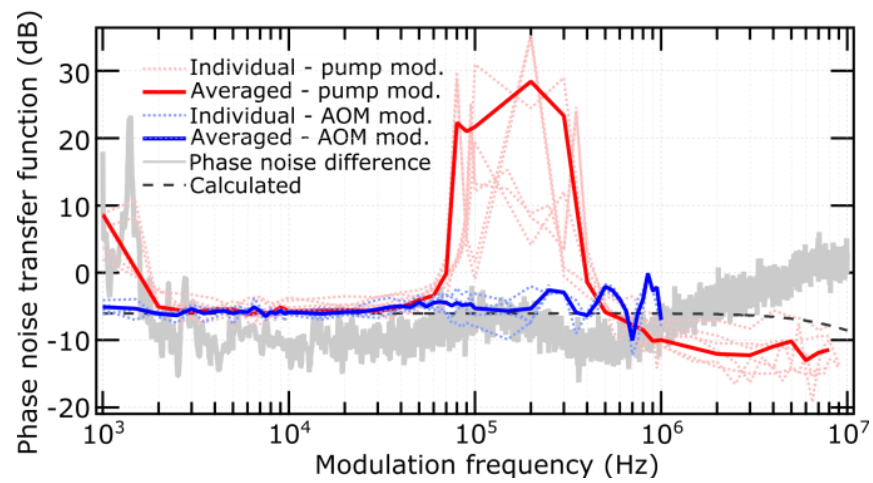

Fig. 6. Measured $f_{0}$ phase noise transfer functions measured via pump power modulation (red traces) and AOM modulation (blue traces). The gray line is the phase noise difference calculated from Fig. 3 and the dashed black line shows the ideal transfer function of a monolithic DOPO according to Ref. [24].

All individual transfer function measurements together with the average for both modulation methods are presented in Fig. 6 together with the analytical phase noise transfer function of a monolithic DOPO according to Ref. [24]. The calculated transfer function exhibits a $6 \mathrm{~dB}$ phase noise reduction until the cavity linewidth limit of $5 \mathrm{MHz}$ for our OPO cavity. The transfer functions for both modulation methods agree well in the Fourier frequency range $1 \mathrm{kHz}$ to $\sim 50 \mathrm{kHz}$ and show a $-6 \mathrm{~dB}$ noise reduction, as expected for a coherent frequency divider. In the range of $\sim 50 \mathrm{kHz}$ to $\sim 500 \mathrm{kHz}$ where the pump power modulation method suffers 
from the lasers relaxation oscillation, the AOM modulation method confirms the $-6 \mathrm{~dB}$ noise reduction. Above $\sim 200 \mathrm{kHz}$, the transfer function measured with AOM modulation also shows some fluctuations which are most likely caused by driving our RF source beyond its specified modulation frequency limit of $100 \mathrm{kHz}$. In fact, we observed a carrier frequency shift of the modulated RF source when the modulation frequency was above $200 \mathrm{kHz}$. Above $\sim 500 \mathrm{kHz}$, the transfer function measured via pump power modulation is negative again and even below the analytical $-6 \mathrm{~dB}$ line. This, however, might be a measurement artifact arising from phase to amplitude coupling. As a comparison, the phase noise difference calculated from Fig. 3 is also plotted in the background in Fig. 6. It also shows constantly the phase noise reduction of the OPO until $1 \mathrm{MHz}$. Above $1 \mathrm{MHz}$, in this measurement the phase noise ratio increases, because we are reaching the detector noise floor for the OPO phase noise measurement (compare Fig 3.).

In summary, we presented a measurement of the $f_{0}$ phase noise transfer function from the driving frequency comb to the output of a DOPO. We find that the DOPO has a $6 \mathrm{~dB}$ phase noise reduction with respect to the pump comb in the Fourier frequency range from $1 \mathrm{kHz}$ to $200 \mathrm{kHz}$ and a substantial phase-noise reduction from $500 \mathrm{kHz}$ to $8 \mathrm{MHz}$ which constitutes the full frequency range accessible with our experimental setup. These measurements prove that a synchronously pumped OPO operated at degeneracy can serve as an ideal coherent frequency divider and constitutes a well-suited scheme for the generation of low-noise FCs in the important mid-IR spectral region.

\section{References}

1. T. Udem, R. Holzwarth, and T. W. Hänsch, Nature 416, 233-7 (2002).

2. A. Foltynowicz, P. Masłowski, T. Ban, F. Adler, K. C. Cossel, T. C. Briles, and J. Ye, Faraday Discuss. 150, 23-31 (2011).

3. A. Schliesser, N. Picqué, and T. W. Hänsch, Nature Photonics 6, 440-449 (2012).

4. A. Hugi, G. Villares, S. Blaser, H. C. Liu, and J. Faist, Nature 492, 229-233 (2012).

5. P. Del'Haye, A. Schliesser, O. Arcizet, T. Wilken, R. Holzwarth, and T. J. Kippenberg, Nature 450, 1214-1217 (2007).

6. C. Fischer and M. W. Sigrist, Topics in Applied Physics No. 89 (Springer Berlin Heidelberg, 2003), pp. 99-143.

7. A. Ruehl, A. Gambetta, I. Hartl, M. E. Fermann, K. S. E. Eikema, and M. Marangoni, Opt. Lett. 37, 2232 (2012).

8. Y. Kobayashi, K. Torizuka, A. Marandi, R. L. Byer, R. A. McCracken, Z. Zhang, and D. T. Reid, Journal of Optics 17, 94010 (2015).

9. F. Adler, K. C. Cossel, M. J. Thorpe, I. Hartl, M. E. Fermann, and J. Ye, Opt. Lett. 34, 1330-1332 (2009).

10. N. Leindecker, A. Marandi, R. L. Byer, and K. L. Vodopyanov, Opt. Express 19, 6296-6302 (2011).

11. K. F. Lee, J. Jiang, C. Mohr, J. Bethge, M. E. Fermann, N. Leindecker, K. L. Vodopyanov, P. G. Schunemann, and I. Hartl, Opt. Lett. 38, 1191-1193 (2013).

12. S. T. Wong, T. Plettner, K. L. Vodopyanov, K. Urbanek, M. Digonnet, and R. L. Byer, Opt. Lett. 33, 1896-1898 (2008).

13. N. Leindecker, A. Marandi, R. L. Byer, and K. L. Vodopyanov, Opt. Express 19, 6296-302 (2011).

14. N. Leindecker, A. Marandi, R. L. Byer, K. L. Vodopyanov, J. Jiang, I. Hartl, M. Fermann, and P. G. Schunemann, Opt. Express 20, 7046 (2012).

15. A. Marandi, N. Leindecker, V. Pervak, R. L. Byer, and K. L. Vodopyanov, Opt. Express 20, 8242-8249 (2012).

16. K. F. Lee, C. Mohr, J. Jiang, P. G. Schunemann, K. L. Vodopyanov, and M. E. Fermann, Opt. Express 23, 26596 (2015)

17. J. H. Sun, B. J. S. Gale, and D. T. Reid, Opt. Lett. 32, 1414-1416 (2007).
18. T. I. Ferreiro, J. Sun, and D. T. Reid, Opt. Lett. 35, 1668 (2010).

19. C. D. Nabors, S. T. Yang, T. Day, and R. L. Byer, J. Opt. Soc. Am. B 7, 815820 (1990).

20. A. Ruehl, M. J. Martin, K. C. Cossel, L. Chen, H. McKay, B. Thomas, C. Benko, L. Dong, J. M. Dudley, M. E. Fermann, I. Hartl, and J. Ye, Phys. Rev. A 84, 011806 (2011).

21. L. Fu, B. K. Thomas, and L. Dong, Opt. Express 16, 19629 (2008).

22. C. Wan, P. Li, A. Chong, A. Ruehl, and I. Hartl, CLEO Europe, paper CF_1_6 (2015).

23. N. R. Newbury and W. C. Swann, J. Opt. Soc. Am. B 24, 1756 (2007).

24.P. D. Drummond, K. J. McNeil, and D. F. Walls, Opt. Acta. 27, 321-335 (1981). 


\section{References}

1. T. Udem, R. Holzwarth, and T. W. Hänsch, "Optical frequency metrology." Nature 416, 233-7 (2002).

2. A. Foltynowicz, P. Masłowski, T. Ban, F. Adler, K. C. Cossel, T. C. Briles, and J. Ye, "Optical frequency comb spectroscopy," Faraday Discuss. 150, 2331 (2011).

3. A. Schliesser, N. Picqué, and T. W. Hänsch, "Mid-infrared frequency combs," Nature Photonics 6, 440-449 (2012).

4. A. Hugi, G. Villares, S. Blaser, H. C. Liu, and J. Faist, "Mid-infrared frequency comb based on a quantum cascade laser," Nature 492, 229233 (2012).

5. P. Del'Haye, A. Schliesser, O. Arcizet, T. Wilken, R. Holzwarth, and T. J. Kippenberg, "Optical frequency comb generation from a monolithic microresonator," Nature 450, 1214-1217 (2007).

6. C. Fischer and M. W. Sigrist, "Mid-IR Difference Frequency Generation," in Solid-State Mid-Infrared Laser Sources, D. I. T. Sorokina and D. K. L. Vodopyanov, eds., Topics in Applied Physics No. 89 (Springer Berlin Heidelberg, 2003), pp. 99-143.

7. A. Ruehl, A. Gambetta, I. Hartl, M. E. Fermann, K. S. E. Eikema, and M. Marangoni, "Widely-tunable mid-infrared frequency comb source based on difference frequency generation," Optics Letters 37, 2232 (2012).

8. Y. Kobayashi, K. Torizuka, A. Marandi, R. L. Byer, R. A. McCracken, Z. Zhang, and D. T. Reid, "Femtosecond optical parametric oscillator frequency combs," Journal of Optics 17, 94010 (2015)

9. F. Adler, K. C. Cossel, M. J. Thorpe, I. Hartl, M. E. Fermann, and J. Ye, "Phase-stabilized, $1.5 \mathrm{~W}$ frequency comb at 2.8-4.8 um," Optics Letters 34, 1330-1332 (2009).

10. N. Leindecker, A. Marandi, R. L. Byer, and K. L. Vodopyanov, "Broadband degenerate OPO for mid-infrared frequency comb generation," Optics Express 19, 6296-6302 (2011).

11. K. F. Lee, J. Jiang, C. Mohr, J. Bethge, M. E. Fermann, N. Leindecker, K. L. Vodopyanov, P. G. Schunemann, and I. Hartl, "Carrier envelope offset frequency of a doubly resonant, nondegenerate, mid-infrared GaAs optical parametric oscillator," Optics Letters 38, 1191-1193 (2013).

12. S. T. Wong, T. Plettner, K. L. Vodopyanov, K. Urbanek, M. Digonnet, and R. L. Byer, "Self-phase-locked degenerate femtosecond optical parametric oscillator." Optics Letters 33, 1896-1898 (2008).

13. N. Leindecker, A. Marandi, R. L. Byer, and K. L. Vodopyanov, "Broadband degenerate OPO for mid-infrared frequency comb generation." Optics Express 19, 6296-302 (2011).

14. N. Leindecker, A. Marandi, R. L. Byer, K. L. Vodopyanov, J. Jiang, I. Hartl, M. Fermann, and P. G. Schunemann, "Octave-spanning ultrafast OPO with 2.6-6.1 $\mu \mathrm{m}$ instantaneous bandwidth pumped by femtosecond Tm-fiber laser," Optics Express 20, 7046 (2012).

15. A. Marandi, N. Leindecker, V. Pervak, R. L. Byer, and K. L. Vodopyanov, "Coherence properties of a broadband femtosecond mid-IR optical parametric oscillator operating at degeneracy." Optics Express 20, 82428249 (2012)

16. K. F. Lee, C. Mohr, J. Jiang, P. G. Schunemann, K. L. Vodopyanov, and M. E. Fermann, "Midinfrared frequency comb from self-stable degenerate GaAs optical parametric oscillator." Optics Express 23, 26596 (2015).

17.J. H. Sun, B. J. S. Gale, and D. T. Reid, "Composite frequency comb spanning 0.4-2.4 $\mu \mathrm{m}$ from a phase-controlled femtosecond Ti: sapphire laser and synchronously pumped optical parametric oscillator," Optics Letters 32, 1414-1416 (2007).

18. T. I. Ferreiro, J. Sun, and D. T. Reid, "Locking the carrier-envelope-offset frequency of an optical parametric oscillator without $f-2 f$ selfreferencing," Optics Letters 35, 1668 (2010).

19. C. D. Nabors, S. T. Yang, T. Day, and R. L. Byer, "Coherence properties of a doubly resonant monolithic optical parametric oscillator." Journal of the Optical Society of America B 7, 815-820 (1990).

20. A. Ruehl, M. J. Martin, K. C. Cossel, L. Chen, H. McKay, B. Thomas, C. Benko, L. Dong, J. M. Dudley, M. E. Fermann, I. Hartl, and J. Ye,
"Ultrabroadband coherent supercontinuum frequency comb," Physical Review A 84, 011806 (2011).

21. L. Fu, B. K. Thomas, and L. Dong, "Efficient supercontinuum generations in silica suspended core fibers," Optics Express 16, 19629 (2008).

22. C. Wan, P. Li, A. Chong, A. Ruehl, and I. Hartl, "Carrier-Envelope Offset Frequencies in Doubly-Resonant Synchronously Pumped Optical Parametric Oscillators," in 2015 European Conference on Lasers and Electro-Optics - European Quantum Electronics Conference, (Optical Society of America, 2015), paper CF_1_6.

23. N. R. Newbury and W. C. Swann, "Low-noise fiber-laser frequency combs (Invited)," Journal of the Optical Society of America B 24, 1756 (2007).

24.P. D. Drummond, K. J. McNeil, and D. F. Walls, "Non-equilibrium transitions in sub/second harmonic generation I. Semiclassical theory." Optica Acta 27, 321-335 (1981). 